\title{
Uso de Open Journal System en revistas científicas peruanas
}

\section{Using Open Journal Systems in Peruvian scientific journals}

\author{
Victoria Yance-Yupari* \\ Escuela Profesional de Psicología, \\ Universidad de San Martín de Porres, Perú
}

\section{Resumen}

El uso del Open Journal Systems en el Perú se ha incrementado en la difusión y visibilidad de revistas científicas de acceso abierto. Conocer e indagar el uso del software en las revistas publicadas por universidades peruanas es necesario para identificar la vigencia y actividad en la que se encuentran. Este, es un estudio exploratorio que tiene una muestra conformada por 54 universidades peruanas, en la que se encontró 324 títulos, que evidenció la totalidad de revistas en acceso abierto; $71 \%$ son publicadas por universidades particulares; 27 títulos no tiene ninguna publicación en la plataforma; la versión 2.0 es utilizada por el 73\%; la versión 3.0 la utilizan solo 74 revistas.

Palabras clave: Open Journal System, OJS, revistas científicas, Perú.

\begin{abstract}
The use of Open Journal Systems in Peru is recent, and its dissemination and visibility in scientific journals are increasing. Knowing and investigating the use of the software in journals published by Peruvian universities are necessary to identify validity and activity in which they are. In the exploratory study, the sample consisted of 54 Peruvian universities. Three hundred twentyfour (324) that evidenced the totality of open access journals, out of which $71 \%$ were published by private universities, $27 \mathrm{did}$ not have publications on the
\end{abstract}

Este es un artículo Open Access bajo la licencia Creative Commons Atribución-NoComercial-Compartirlgual 4.0 
platform, $73 \%$ used OJS version 2.0 , and only 74 were using the new version 3.0.

Keywords: Open Journal Systems, OJS, scientific journals, Peru.

\section{Introducción}

La comunidad científica siempre ha tenido la necesidad de publicar, difundir y dar a conocer sus hallazgos a la sociedad, con cierta medida, a través de la universidad, institución que promueve la investigación, así como la formación de investigadores. Estas entidades son las principales editoras de publicaciones científicas, siendo las revistas científicas el medio de difusión más utilizado por los investigadores para divulgar sus estudios y otros materiales bibliográficos como libros y boletines especializados (Acevedo, 1997; Chavarro, Tang, \& Ràfols, 2017; Gómez, Jiménez, \& Moreles, 2014; Piedra \& Martínez, 2007).

La necesidad de pasar de la revista impresa a la revista electrónica surge en la última década. Ante esta transición se han desarrollado nuevas tecnologías que son útiles para la difusión y visibilidad de las investigaciones, así como para la comunicación entre editores y autores.

Frente a esta realidad, los responsables de las revistas científicas, en especial las editadas por las universidades, indagaron en las propuestas para la edición y publicación de estas. Uno de los primeros problemas que encontraron los equipos editoriales es el software o programas a utilizar para difundir las investigaciones (Edgar \& Willinsky, 2010; Guédon, 1999; Martín \& Merlo, 2003; Melero \& Abad, 2008; Willinsky, 2005).

Diversos estudios reportaron la necesidad de contar con uno o varios softwares para la migración del papel al formato electrónico. Melero y Abad (2008) explican que hay diferentes softwares (OJS, HyperJournal, DPubS y SOPS) que se elaboraron para la transición del desarrollo de la revista impresa a la electrónica, utilizando la web como soporte. Estivill, Abadal, Franganillo, Gascón y Rodríguez (2007) encontraron que diversas instituciones españolas utilizaban diferentes tipos de softwares para la administración de revistas (p. ej., OJS, Dspace, Fedora, etc.). Laudun (2012), menciona 13 software libres que permiten gestionar una revista (p. ej., Ambra, CLEO, Diva, E-Journal y otros). 
Es así que, uno de los programas desarrollados para facilitar la transición del formato impreso al digital fue el HyperJournal (Barbera \& Pirro, 2015) este es un software de código libre que permite administrar la gestión editorial de una revista. También tenemos el DPubS. Digital Publishing System, desarrollado por Cornell University Library (2008), su última actualización es del 2007 con la versión 2.1. Otro programa que se desarrolló para la gestión editorial es el SOPS (SciX Open Publishing Services), creado por University of Ljubljana (2004), solo lanzaron la versión 1.0, actualmente ya no están activos o hay pocas revistas que los usan.

En paralelo, Public Knowledge Project (PKP), desarrolló el Open Journal Systems (OJS), desde su lanzamiento como software libre en 2002, era el único que permitía administrar la gestión editorial y difundir las publicaciones de las revistas científicas (Willinsky, 2005). El OJS es un programa gratuito que permite administrar la gestión editorial de una revista; sirve como plataforma de difusión de los estudios que se publican y de plataforma intermediaria entre los editores, autores y bases de datos sobre los procesos editoriales y otras dudas del ciclo editorial, reemplazando a los correos. Desde su aparición a la actualidad se han lanzado 59 mejoras y nuevas versiones (ver Tabla 1).

PKP contabilizó hasta el año 2017 más de 9253 revistas que utilizan el OJS a nivel mundial; y en Latinoamérica y Caribe 2840 (PKP, 2018b). En el Perú se registró 78 revistas peruanas que utilizaban el OJS (Figura 1).

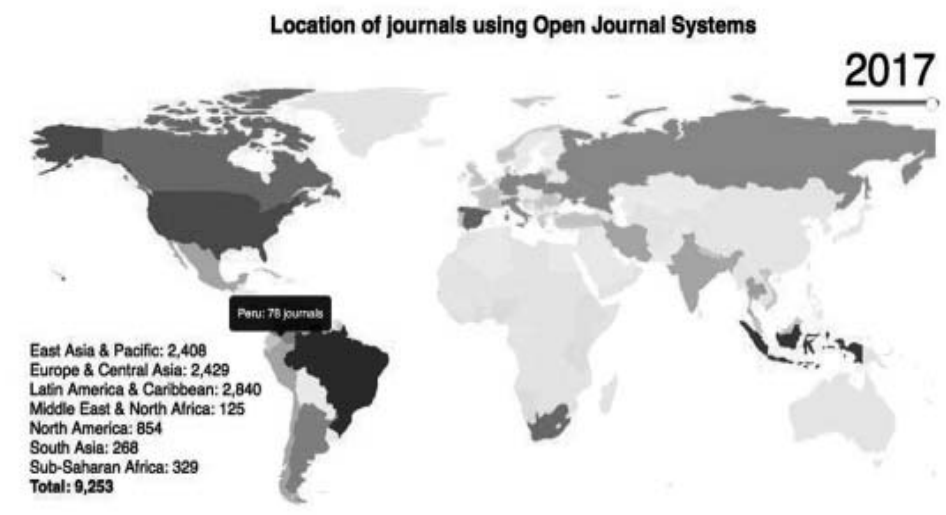

Figura 1. Location of journals using Open Journal Systems (Public Knowledge Project, 2018). 
Tabla 1

Versiones del OJS publicados por Public Knowledge Project

\begin{tabular}{|c|c|c|c|}
\hline & OJS 1 & OJS 2 & OJS 3 \\
\hline $\begin{array}{l}\text { Versiones } \\
\text { del } 2002 \text { al } 2018\end{array}$ & $\begin{array}{l}1.1 .10 \\
1.1 .9 \\
1.1 .8 \\
1.1 .7 \\
1.1 .6 \\
1.1 .5 \\
1.1 \\
1.0 .1 \\
1.0\end{array}$ & $\begin{array}{l}2.4 .8-3 \\
2.4 .8-2 \\
2.4 .8-1 \\
2.4 .8 \\
2.4 .7-1 \\
2.4 .7 \\
2.4 .6 \\
2.4 .5 \\
2.4 .4-1 \\
2.4 .4-0 \\
2.4 .3 \\
2.4 .2 \\
2.4 .1 \\
2.4 .0 \\
2.3 .8 \\
2.3 .7 \\
2.3 .6 \\
2.3 .5 \\
2.3 .4 \\
2.3 .3-3 \\
2.3 .3-2 \\
2.3 .3-1 \\
2.3 .3 \\
2.3 .1-2 \\
2.3 .0 \\
2.2 .4 \\
2.2 .3 \\
2.2 .2 \\
2.2 \\
2.1 .1 \\
2.1 \\
2.0 .2-1 \\
2.0 .1 \\
2.0\end{array}$ & $\begin{array}{l}3.1 .1-2 \\
3.1 .1-1 \\
3.1 .0-1 \\
3.1 .0 \\
3.0 .2 \\
3.0 .1 \\
3.0 .0\end{array}$ \\
\hline Total & 9 & 34 & 7 \\
\hline
\end{tabular}

Nota: Public Knowledge Project (PKP), 2018a; Willinsky, 2005. 
En Latinoamérica el uso del OJS es reciente, especialmente en la última década del presente siglo, siendo liderado por Brasil (Alhuay \& Bautista, 2014; Delgado, 2014; Edgar \& Willinsky, 2010; López, Torréns, Viloria, \& Ramírez, 2012). El uso del OJS permitió incrementar el acceso y visibilidad de las revistas latinoamericanas que se encontraban excluidas, inaccesibles o desconocidas en la comunidad científica (Polanco-Cortés \& Garro, 2013).

En el Perú, recién en el 2010 se inicia la aplicación del software OJS en revistas peruanas; las primeras fueron las revistas editadas por la Universidad Nacional Mayor de San Marcos (Santillán-Aldama, 2010; Quispe-Gerónimo, Santillán-Aldana, \& Balcazar-Tafur, 2013; Universidad Nacional Mayor de San Marcos, 2010). En estudios recientes, Chávez-Sánchez y Estrada-Cuzcano (2016), encontraron 25 universidades peruanas que utilizan OJS en sus sistemas de gestión editorial, con un total de 223 títulos de revistas. Santillán-Aldana et al. (2017) identificaron 138 revistas que utilizan el programa.

El objetivo de la presente investigación es conocer cuántas revistas científicas editadas por universidades peruanas usan el OJS en la actualidad y su presencia en la web.

\section{Metodología}

Se realizó un estudio exploratorio (Salkind, 1999) para conocer e identificar el uso de software OJS por las revistas científicas editadas por universidades peruanas. La población la constituyeron 143 universidades peruanas registradas (SUNEDU, agosto 2018), siendo 51 nacionales y 92 privadas. La muestra, estuvo conformada por 54 universidades del Perú (24 nacionales y 30 privadas). Se utilizó un muestreo aleatorio simple (Hernández et al., Fernandez, \& Baptista, 2014) y se aplicó estadísticos descriptivos (porcentajes).

\section{Procedimiento}

Los datos fueron recogidos de los portales de revistas y páginas webs de las universidades peruanas mediante búsquedas realizadas en Google Scholar, Google y Microsoft Academic. Las palabras clave de búsqueda fueron: revistas, revistas peruanas, universidad, OJS y Perú. Se indagó sobre el tipo de versión 
de OJS que utilizan las revistas, título de las revista, número de publicación (volumen y/o número) y tipo de universidad.

Se encontró 351 revistas en la revisión de cada portal, se excluyó 27 títulos que no tenían ningún artículo publicado en el portal de OJS y se analizó solo 324 journals.

Asimismo, se elaboró una base de datos en Excel con la información obtenida entre los meses de mayo hasta agosto del 2018.

\section{Resultados}

En la revisión de los tipos de OJS utilizados por las revistas, se encontró que la mayoría utiliza la versión 2 (73\%) y solo $23 \%$ la versión 3 (Figura 2).

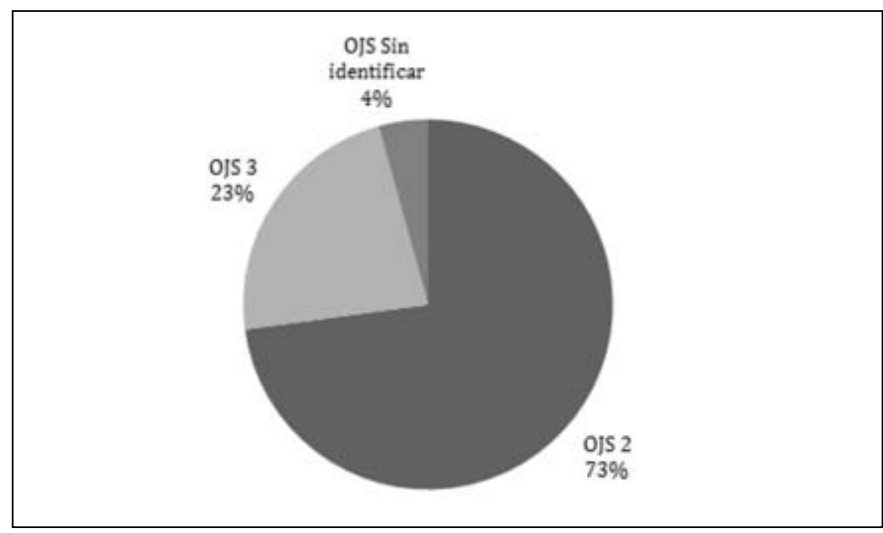

Figura 2. Versiónes de OJS utilizados.

La versión, 2.4.8.1 (20\%) es la más utilizada. Actualmente, solo 74 revistas publicadas ya utilizan las ultimas versiones (3.0) publicadas por PKP, a pesar que ya se encontraba disponible desde fines del 2016 (Tabla 2).

Todas las revistas editadas por las universidades peruanas encontradas, son de acceso abierto. El porcentaje de las que son publicadas por instituciones nacionales es menor (29\%) que las editadas por instituciones particulares $(71 \%)$ (Figura 3). 


\section{Tabla 2}

Tipos de versión del OJS que usan las revistas peruanas

\begin{tabular}{crc}
\hline Versiones de OJS & C & $\%$ \\
\hline 2.3 .8 .0 & 5 & $2 \%$ \\
2.4 .1 .0 & 5 & $2 \%$ \\
2.4 .3 .0 & 5 & $2 \%$ \\
2.4 .4 .0 & 8 & $2 \%$ \\
2.4 .4 .1 & 14 & $4 \%$ \\
2.4 .6 .0 & 41 & $13 \%$ \\
2.4 .7 .1 & 8 & $2 \%$ \\
2.4 .8 .0 & 51 & $16 \%$ \\
2.4 .8 .1 & 64 & $20 \%$ \\
2.4 .8 .2 & 15 & $5 \%$ \\
2.4 .8 .3 & 20 & $6 \%$ \\
3.0 .2 .0 & 29 & $9 \%$ \\
3.1 .0 .1 & 21 & $6 \%$ \\
3.1 .1 .0 & 16 & $5 \%$ \\
3.1 .1 .2 & 8 & $2 \%$ \\
OJS Sin identificar & 14 & $4 \%$ \\
\hline
\end{tabular}

Nota: $\mathrm{C}=$ cantidad; $\%=$ porcentaje.

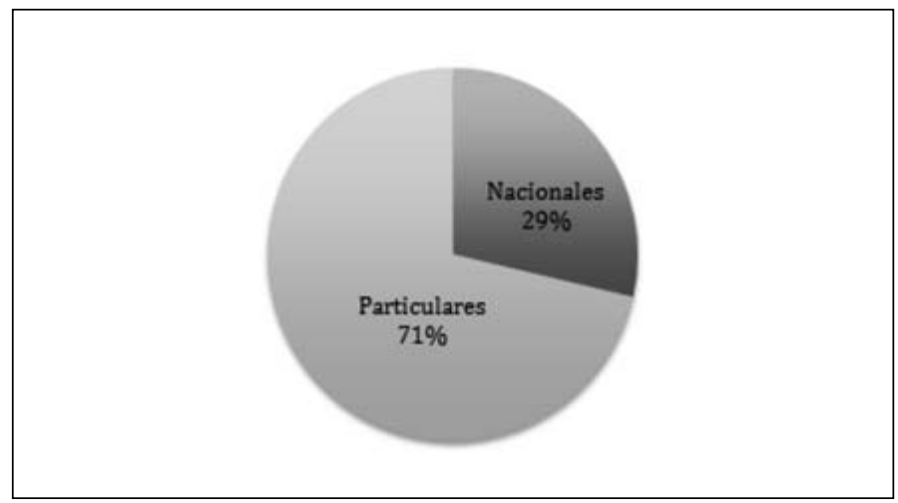

Figura 3. Revistas publicadas por tipo de universidad.

En la exclusión de datos se identificó que no todos los títulos publicados con el sofware OJS en la red, tienen publicados números o volúmenes en sus portales. Se encontró 27 títulos de revistas que solo registran el nombre de la revista, pero no contienen artículos (Tabla 3). 
Tabla 3

Títulos de revistas sin publicaciones en el OJS

\begin{tabular}{|c|c|c|c|c|c|}
\hline OJS & REVISTA & NOMBRE UNIVERSIDAD & $U$ & C & URL \\
\hline 3.1.0.1 & $\begin{array}{l}\text { MINDS/Revista Científica Digital } \\
\text { de Est. de Psicología }\end{array}$ & $\begin{array}{l}\text { Universidad César } \\
\text { Vallejo }\end{array}$ & $P$ & 0 & $\begin{array}{l}\text { http://revistas.ucv.edu.pe/index.php/ } \\
\text { Minds }\end{array}$ \\
\hline 2.4.8.1 & Peruvian journal of computing & $\begin{array}{l}\text { Universidad Nacional de } \\
\text { Trujillo }\end{array}$ & $\mathrm{N}$ & 0 & $\begin{array}{l}\text { http://revistas.unitru.edu.pe/ } \\
\text { index.php/PERJOUCOMP }\end{array}$ \\
\hline 2.4.8.1 & $\begin{array}{l}\text { Revista de investigación } \\
\text { matemática }\end{array}$ & $\begin{array}{l}\text { Universidad Nacional de } \\
\text { Trujillo }\end{array}$ & $\mathrm{N}$ & 0 & $\begin{array}{l}\text { http://revistas.unitru.edu.pe/ } \\
\text { index.php/BOCIENMAT }\end{array}$ \\
\hline 2.4.8.1 & $\begin{array}{l}\text { Revista de investigación } \\
\text { estadística }\end{array}$ & $\begin{array}{l}\text { Universidad Nacional de } \\
\text { Trujillo }\end{array}$ & $\mathrm{N}$ & 0 & $\begin{array}{l}\text { http://revistas.unitru.edu.pe/ } \\
\text { index.php/REDIES }\end{array}$ \\
\hline 2.4.8.1 & Revista digital de Informática & $\begin{array}{l}\text { Universidad Nacional de } \\
\text { Trujillo }\end{array}$ & $\mathrm{N}$ & 0 & $\begin{array}{l}\text { http://revistas.unitru.edu.pe/ } \\
\text { index.php/Revistadelnformatica }\end{array}$ \\
\hline 2.4.8.1 & $\begin{array}{l}\text { Revista científica de ciencias } \\
\text { físicas }\end{array}$ & $\begin{array}{l}\text { Universidad Nacional de } \\
\text { Trujillo }\end{array}$ & $\mathrm{N}$ & 0 & $\begin{array}{l}\text { http://revistas.unitru.edu.pe/ } \\
\text { index.php/rccff }\end{array}$ \\
\hline 2.4.8.1 & Visión industrial & $\begin{array}{l}\text { Universidad Nacional de } \\
\text { Trujillo }\end{array}$ & $\mathrm{N}$ & 0 & $\begin{array}{l}\text { http://revistas.unitru.edu.pe/ } \\
\text { index.php/RINGIND }\end{array}$ \\
\hline 2.4.8.1 & $\begin{array}{l}\text { Facultad de Ciencias } \\
\text { Económicas-Contabilidad }\end{array}$ & $\begin{array}{l}\text { Universidad Nacional de } \\
\text { Trujillo }\end{array}$ & $\mathrm{N}$ & 0 & $\begin{array}{l}\text { http://revistas.unitru.edu.pe/ } \\
\text { index.php/contabilidad }\end{array}$ \\
\hline 2.4.8.1 & $\begin{array}{l}\text { Revista de la Escuela de } \\
\text { Ingeniería Agrícola }\end{array}$ & $\begin{array}{l}\text { Universidad Nacional de } \\
\text { Trujillo }\end{array}$ & $\mathrm{N}$ & 0 & $\begin{array}{l}\text { http://revistas.unitru.edu.pe/ } \\
\text { index.php/RIA/issue/archive }\end{array}$ \\
\hline 2.4.8.1 & $\begin{array}{l}\text { AMAUTA. Revista Científica } \\
\text { de la Facultad de Educación } \\
\text { y Ciencias de la } \\
\text { Comunicación }\end{array}$ & $\begin{array}{l}\text { Universidad Nacional de } \\
\text { Trujillo }\end{array}$ & $\mathrm{N}$ & 0 & $\begin{array}{l}\text { http://revistas.unitru.edu.pe/ } \\
\text { index.php/RAMAUTA }\end{array}$ \\
\hline 2.4.8.1 & $\begin{array}{l}\text { NOS OTROS. Revista } \\
\text { Científica de la Facultad de } \\
\text { Ciencias Sociales }\end{array}$ & $\begin{array}{l}\text { Universidad Nacional de } \\
\text { Trujillo }\end{array}$ & $\mathrm{N}$ & 0 & $\begin{array}{l}\text { http://revistas.unitru.edu.pe/ } \\
\text { index.php/facccsoc }\end{array}$ \\
\hline 2.4.8.1 & $\begin{array}{l}\text { Revista de la Facultad de } \\
\text { Derecho y Ciencias Políticas }\end{array}$ & $\begin{array}{l}\text { Universidad Nacional de } \\
\text { Trujillo }\end{array}$ & $\mathrm{N}$ & 0 & $\begin{array}{l}\text { http://revistas.unitru.edu.pe/ } \\
\text { index.php/facderccpp }\end{array}$ \\
\hline 2.4.8.1 & $\begin{array}{l}\text { Revista Científica de la } \\
\text { Facultad de Ingeniería } \\
\text { Química }\end{array}$ & $\begin{array}{l}\text { Universidad Nacional de } \\
\text { Trujillo }\end{array}$ & $\mathrm{N}$ & 0 & $\begin{array}{l}\text { http://revistas.unitru.edu.pe/ } \\
\text { index.php/facingquim }\end{array}$ \\
\hline 2.4.8.1 & $\begin{array}{l}\text { Revista Científica de la } \\
\text { Facultad de Ingeniería }\end{array}$ & $\begin{array}{l}\text { Universidad Nacional de } \\
\text { Trujillo }\end{array}$ & $\mathrm{N}$ & 0 & $\begin{array}{l}\text { http://revistas.unitru.edu.pe/ } \\
\text { index.php/facing }\end{array}$ \\
\hline
\end{tabular}




\begin{tabular}{|c|c|c|c|c|c|}
\hline OJS & REVISTA & NOMBRE UNIVERSIDAD & $U$ & C & URL \\
\hline 2.4.8.1 & $\begin{array}{l}\text { Revista de Autoevaluación y } \\
\text { Acreditación }\end{array}$ & $\begin{array}{l}\text { Universidad Nacional de } \\
\text { Trujillo }\end{array}$ & $\mathrm{N}$ & 0 & $\begin{array}{l}\text { http://revistas.unitru.edu.pe/ } \\
\text { index.php/RAA }\end{array}$ \\
\hline 2.4.8.1 & $\begin{array}{l}\text { Secundaria - Historia y } \\
\text { Geografía }\end{array}$ & $\begin{array}{l}\text { Universidad Nacional de } \\
\text { Trujillo }\end{array}$ & $\mathrm{N}$ & 0 & $\begin{array}{l}\text { http://revistas.unitru.edu.pe/ } \\
\text { index.php/RSHYG }\end{array}$ \\
\hline 2.4.8.1 & $\begin{array}{l}\text { Secundaria - Ciencias } \\
\text { Matemáticas }\end{array}$ & $\begin{array}{l}\text { Universidad Nacional de } \\
\text { Trujillo }\end{array}$ & $\mathrm{N}$ & 0 & $\begin{array}{l}\text { http://revistas.unitru.edu.pe/ } \\
\text { index.php/RSCCMM }\end{array}$ \\
\hline 2.4.8.1 & $\begin{array}{l}\text { Secundaria - Ciencias } \\
\text { Naturales }\end{array}$ & $\begin{array}{l}\text { Universidad Nacional de } \\
\text { Trujillo }\end{array}$ & $\mathrm{N}$ & 0 & $\begin{array}{l}\text { http://revistas.unitru.edu.pe/ } \\
\text { index.php/RSCCNN }\end{array}$ \\
\hline 2.4.8.1 & $\begin{array}{l}\text { Secundaria - Filosofía, } \\
\text { Psicología y Ciencias Sociales }\end{array}$ & $\begin{array}{l}\text { Universidad Nacional de } \\
\text { Trujillo }\end{array}$ & $\mathrm{N}$ & 0 & $\begin{array}{l}\text { http://revistas.unitru.edu.pe/ } \\
\text { index.php/RSFPCCSS }\end{array}$ \\
\hline 2.4.6.0 & $\begin{array}{l}\text { Cantua. Revista de Ciencias } \\
\text { Biológicas }\end{array}$ & $\begin{array}{l}\text { Universidad Nacional de } \\
\text { San Antonio Abad del Cusco }\end{array}$ & $\mathrm{N}$ & 0 & $\begin{array}{l}\text { http://revistas.unsaac.edu.pe/ } \\
\text { index.php/RCCB }\end{array}$ \\
\hline 2.4.6.0 & Acta Médica de la UNSA & $\begin{array}{c}\text { Universidad Nacional de San } \\
\text { Agustín de Arequipa }\end{array}$ & $\mathrm{N}$ & 0 & $\begin{array}{l}\text { http://revistas.unsa.edu.pe/ } \\
\text { index.php/Gaceta }\end{array}$ \\
\hline 2.4.6.0 & Supra Juris & $\begin{array}{l}\text { Universidad de San Martín de } \\
\text { Porres }\end{array}$ & $\mathrm{P}$ & 0 & $\begin{array}{l}\text { https://uww.aulavirtualusmp. pe/ojs/ } \\
\text { index.php/SJ }\end{array}$ \\
\hline 2.4.8.0 & $\begin{array}{l}\text { Sociedad y Desarrollo } \\
\text { Subnacional }\end{array}$ & $\begin{array}{l}\text { Universidad Nacional Pedro } \\
\text { Ruiz Gallo }\end{array}$ & $\mathrm{N}$ & 0 & $\begin{array}{l}\text { http://revistas.unprg.edu.pe/ } \\
\text { openjournal/index.php/sociedad/ } \\
\text { issue/archive }\end{array}$ \\
\hline 2.4.8.0 & $\begin{array}{l}\text { Revista en Ciencia y } \\
\text { Tecnología de Alimentos } \\
\text { Funcionales }\end{array}$ & $\begin{array}{l}\text { Universidad Nacional Pedro } \\
\text { Ruiz Gallo }\end{array}$ & $\mathrm{N}$ & 0 & $\begin{array}{l}\text { http://revistas.unprg.edu.pe/ } \\
\text { openjournal/index.php/cytaf }\end{array}$ \\
\hline 3.1.1.2 & Revista Mast'ariy & $\begin{array}{l}\text { Universidad Andina del } \\
\text { Cusco }\end{array}$ & $P$ & 0 & $\begin{array}{l}\text { http://revistas.uandina.edu.pe/ } \\
\text { index.php/mastariy }\end{array}$ \\
\hline 3.1.1.2 & $\begin{array}{l}\text { UAC Revista de la Facultad } \\
\text { de Derecho }\end{array}$ & $\begin{array}{l}\text { Universidad Andina del } \\
\text { Cusco }\end{array}$ & $\mathrm{P}$ & 0 & $\begin{array}{l}\text { http://revistas. uandina.edu.pe/ } \\
\text { index.php/UACD/issue/archive }\end{array}$ \\
\hline 3.1.1.2 & Arquitectura Andina & $\begin{array}{l}\text { Universidad Andina del } \\
\text { Cusco }\end{array}$ & $P$ & 0 & $\begin{array}{l}\text { http://revistas. uandina.edu.pe/ } \\
\text { index.php/ARAN/issue/archive }\end{array}$ \\
\hline
\end{tabular}

Nota: $\mathrm{U}=$ tipo de universidad; $\mathrm{C}=$ publicaciones. 


\section{Discusión}

El uso del software OJS a nivel mundial ha permitido identificar el interés creciente en utilizar el programa por las revistas científicas, editadas por universidades peruanas como plataforma de visibilidad y gestión editorial. Diversos autores propusieron el uso del OJS para la creación de plataformas de revistas con el fin de incentivar la difusión y visibilidad de las investigaciones publicadas (Edgar \& Willinsky, 2010; Polanco-Cortés \& Garro, 2013).

El objetivo de conocer cuántas revistas científicas editadas por universidades peruanas usan el OJS en la actualidad, se justifica por el interés de diversas instituciones educativas en incrementar la producción científica del Perú, para tener mayor presencia en el mundo académico nacional e internacional. Además, este estudio exploratorio es una línea de base para conocer cuántas revistas científicas existen en el Perú que se encuentran o no indexadas en una base de datos, por desconocimiento o desinterés del equipo editorial. No se realizo búsquedas en las bases de acceso abierto como DOAJ, Scielo, Redalyc, Latindex o ROAD, porque limitaría los resultados solo a revistas indexadas y no fue el objetivo del estudio. También, es necesario continuar con el análisis de cada revista, para identificar si cumple o no los criterios o normas editoriales (e.g., ISSN, vigencia, comité editorial, periodicidad y otros).

En los resultados se encontró 324 revistas en acceso abierto que utilizan el software OJS; en comparación con estudios de años anteriores su uso se ha incrementado, esto permite mayor visibilidad y accesibilidad de los estudios publicados en estas revistas peruanas editadas por universidades (ChávezSánchez \& Estrada-Cuzcano, 2016; Laakso et al., 2011; Santillan-Aldama, 2010; Quispe-Geronimo, Santillan-Aldana, \& Balcazar-Tafur, 2013; Santillan-Aldana et al., 2017).

En el Perú la versión más utilizada del OJS es la versión 2.0, a pesar de que la versión 3.0 apareció a fines del 2016, todavía las revistas no han iniciado la migración de una versión a otra para continuar con la gestión editorial. El proceso de migración, no solo implica la descarga e instalación del programa, sino que debe complementarse con capacitaciones permanentes del uso del 
software en la gestión editorial. Esto implica un costo adicional (e.g., horas hombre, equipamiento, etc.) en la edición de una revista científica que muchas universidades no contemplan en su presupuesto (Rozemblum \& Banzato, 2018). Además, es necesaria la participación activa de los editores, autoridades y gestores de información

En el recojo de datos se excluyó 27 títulos de revistas que no tenían publicaciones. Se desconoce, los motivos de publicar los títulos sin ningún número o artículo; esta práctica puede ocurrir: (1) con el fin de separar nombre de la futura publicación; (2) recién se está dando a conocer alguna revista regional o institucional que solo se difundía localmente; y (3) falta de mantenimiento y actualización de la página donde se encuentra la revista.

Esta necesidad de información, de conocer como están desarrollándose las revistas científicas peruanas y la identificación de nuevas revistas editadas por las universidades, surge ante la preocupación de diversas instituciones de educación superior por cumplir con los estándares para acreditarse por la Superintendencia Nacional de Educación Superior Universitaria (2016) y así continuar activas. La proliferación excesiva de revistas científicas editadas por universidades, puede acarrear un problema a futuro como los casos de Colombia y México, que frente al incremento de revistas científicas, están pasando por nuevas evaluaciones (Días, 2017; Ossa \& Cudina, 2016; Vasen \& Lujano, 2017) y provocando el revisticidio (revistas condenadas a desaparecer por no cumplir los criterios de evaluación).

\section{Aspectos éticos}

Los datos de las revistas son de acceso público y no se necesita tener clave de acceso. Los datos disponibles en los portales peruanos de revistas, no se contradicen con lo expuesto en la Ley № 29733 de Protección de Datos Personales. Google Scholar, Google y Microsoft Academic, son buscadores que permiten acceso a artículos, libros y tesis de diferentes repositorios, bases de datos y portales académicos en Open Access. 


\section{Referencias}

Acevedo, J. (1997). ¿Publicar o Patentar? Hacia una Ciencia cada vez más ligada a la Tecnología. Revista Española de Física, 11(2), 8-11. Recuperado de https://www.oei.es/historico/salactsi/acevedo4.htm

Alhuay, J., \& Bautista, L. (2014). El uso de Open Journal Systems y la presencia en Google Scholar de revistas científicas de Bibliotecología de América Latina y El Caribe. Revista Infoacceso, 1(1), 31-36. Recuperado de http://www.revistas.infoacceso.org/index.php/ri/article/view/5/13

Barbera, M., \& Pirro, P. (2015). HyperJournal. Recuperado https://sourceforge.net/projects/hyperjournal/

Cornell University Library. (2008). DPubS. Digital Publishing System. Recuperado de http://dpubs.org/

Chavarro, D., Tang, P., \& Ràfols, I. (2017). Why researchers publish in non-mainstream journals: Training, knowledge bridging, and gap filling. Research Policy, 46(9), 1666-1680. doi: https://doi.org/10.1016/ j.respol.2017.08.002

Chávez-Sánchez, H., \& Estrada-Cuzcano, A. (2016). La gestión de revistas electrónicas en las universidades peruanas. MediaLabUNMSM. Recuperado de http://medialab.letras.unmsm.edu.pe/?p=2257

Delgado, J. E. (2014). Revistas científicas odontológicas de libre acceso en Iberoamérica y Colombia. Revista Facultad de Odontología Universidad de Antioquia, 26(1). Recuperado de http://www.scielo.org.co/ scielo.php?script=sci_arttext\&pid=S0121-246X2014000200009

Díaz, G. (2017). Incertidumbre ante la implementación del nuevo modelo de medición de revistas científicas en Colombia. Tecno Lógicas, 20(38). Recuperado de www.scielo.org.co/pdf/teclo/v20n38/v20n38a01.pdf

Edgar, B. D., \& Willinsky, J. (2010) A survey of scholarly journals using Open Journal Systems. Scholarly and Research Communication, 1. Recuperado de http://src-online.ca/index.php/src/article/view/24/41

Estivill, A., Abadal, E., Franganillo, J., Gascoín, J., \& Rodríguez, J. M. (2007). Uso de metadatos en depositos institucionales españoles: una primera aproximación. En Jornadas Españolas de Documentación. Einformación: integración y rentabilidad en un entorno digital. Fesabid 2007. Santiago de Compostela: FESABID. Recuperado de http://eprints.rclis.org/9666/

Gómez, A., Jiménez, S., \& Moreles, J. (2014). Publicar en revistas científicas, recomendaciones de investigadores de ciencias sociales y humanidades. Revista mexicana de investigación educative, 19(60). Recuperado de http://www.scielo.org.mx/scielo.php?script=sci_arttext\&pid=S1405-66662014000100008

Guédon, Jean-Claude (1999). Surfaces. Electronic journal. Recuperado de https://pum.umontreal.ca/revues/ surfaces/home.html

Guédon, Jean-Claude (2004). The 'green' and 'gold' roads to open access: the case for mixing and matching. Serials Review, 30(4), 315-328. Recuperado de http://eprints.rclis.org/5860/

Hernández, R., Fernández, C., \& Baptista, P. (2014). Metodología de la investigación. México: Mc Graw Hill.

Hernández, F., \& Maquilón, J. (2010). Indicadores de calidad de las revistas científicas y sistema de gestión editorial mediante OJS. Revista de investigación Educativa, 28(1), 13-29. Recuperado de http://revistas.um.es/ rie/article/view/109941

Laakso, M., Welling, P., Bukvova, H., Nyman, L., Björk, B. C., \& Hedlund, T. (2011). The development of open access journal publishing from 1993 to 2009. PloS one, 6(6), e20961. Recuperado de https://journals.plos.org/ plosone/article?id=10.1371/journal. pone.0020961 
Laudun, J. (2012). Free and Open Source Journal Management Software. Recuperado de http://johnlaudun.org/ 20120904-free-and-open-source-journal-management-software/

López, Y., Torréns, R., Viloria, A., \& Ramírez, M. (2012). OJS: Una herramienta de gestión editorial en línea. Estrategias para su adopción en la Universidad de Los Andes. Anuario electrónico de estudios en Comunicación Social Disertaciones, 5(1), 217-228. Recuperado de http://revistas.urosario.edu.co/index.php/ disertaciones/article/view/3895

Martín, J. C., \& Merlo, J. A. (2003). Las revistas electrónicas: características, fuentes de información y medios de acceso. Anales de documentación, 6, 155-186. Recuperado de http://revistas.um.es/analesdoc/article/view/ 1971

Melero, R., \& Abad, F. (2008). Revistas open access: características, modelos económicos y tendencias. BiD: textos universitaris de biblioteconomia $i$ documentació, 20. Recuperado de http://bid.ub.edu/20meler1.htm

Ossa, J. C., \& Cudina, J. N. (2016). Sesenta años de las revistas de psicología en Colombia. Revista Guillermo de Ockham, 14(2), 7-17. doi: http://dx.doi.org/10.21500/22563202.2720

Piedra, Y., \& Martínez, A. (2007). Producción científica. Ciencias de la Información, 38(3), 33-38. Recuperado de http://www.redalyc.org/pdf/1814/181414861004.pdf

Polanco-Cortés, J., \& Garro, M. (2013). Propuesta de modelo centroamericano para la creación de portales nacionales de revistas a través de las universidades estatales. En III Conferencia Internacional de «Acceso abierto, preservación digital y datos científicos» III Conferecia Bibliotecas y Repositorios Digitales de América Latina (BIREDIAL'13) VIII Simposio Internacional de Bibliotecas Digitales (SIBD'13), Ciudad de la Investigación, Universidad de Costa Rica. Recuperado de http://eprints.rclis.org/20568/1/P\%C3\%B3ster\%2064-274-1-PB.pdf

Public Knowledge Project. (2018a). Download. Recuperado de https://pkp.sfu.ca/ojs/ojs_download/

Public Knowledge Project. (2018b). How Many Journals Use OJS? Recuperado de https://pkp.sfu.ca/2015/10/01/ how-many-journals-use-ojs/

Quispe-Gerónimo, C., Santillán-Aldana, J., \& Balcazar-Tafur, D. (2013) Estado del acceso abierto en el Perú 20012013: pequeños pero sostenidos esfuerzos para la comunidad académica peruana. Recuperado de http:// eprints.rclis.org/20462/

Rozemblum, C., \& Banzato, G. (2018). Modelo sustentable de gestión editorial en Acceso Abierto. En III Congreso Internacional de editores de RedALyC. Trujillo: RedALyC. Recuperado de http://congreso.redalyc.org/ocs/ public/congresoEditores/img/presentaciones/CeciliaRozemblum.pdf

Salkind, N. J. (1999). Métodos de investigación. Pearson Educación. 80

Santillán-Aldama, J. (2010). OJS en América Latina. Recuperado http://eprints.rclis.org/14810/1/ ojs_america_latina.pdf

Santillán-Aldana, J., Arakaki, M., de la Vega, A., Calderón-Carranza, M., \& Pacheco-Mendoza, J. (2017). Características generales de las revistas científicas peruanas. Revista Española de Documentación Científica, 40(3), e182. doi: http://dx.doi.org/10.3989/redc.2017.3.1419

Superintendencia Nacional de Educación Superior Universitaria. (2016). El Modelo de Licenciamiento y su Implementación en el Sistema Universitario Peruano. Recuperado de https://www.sunedu.gob.pe/direccionde-licenciamiento-4/condiciones-basicas-de-calidad/

Vase, F., \& Lujano, I. (2017). Sistemas nacionales de clasificación de revistas científicas en América Latina: tendencias recientes e implicaciones para la evaluación académica en ciencias sociales. Revista Mexicana 
de Ciencias Políticas y Sociales, 62(231). Recuperado de https://www.sciencedirect.com/science/article/pii/ S0185191817300430

University of Ljubljana. (2004). SciX Open Publishing Services. Recuperado de http://www.scix.net/sops.htm

Universidad Nacional Mayor de San Marcos. (2010). Edición y publicación de revistas con Open Journal System. Recuperado de http://www.unmsm.edu.pe/noticias/ver/1135

Willinsky, J. (2005). Open Journal Systems: An example of open source software for journal management and publishing. Library Hi Tech, 23(4), 504-519. doi: 10.1108/07378830510636300. Recuperado de http:// psycnet.apa.org/buy/1999-03403-008 